Phase- shi ft ext ract i on from twi ce- nor mal i zed I i ght i ntensi ty changes recorded wi th $r$ andom phase shifts

\begin{tabular}{|l|l|}
\hline 著者 & Adachi Nesaaki, Sunada Sat oshi \\
\hline $\begin{array}{l}\text { j our nal or } \\
\text { publ i cat i on t i t l e }\end{array}$ & Opt i cal Revi ew \\
\hline vol ume & 21 \\
\hline nunber & 5 \\
\hline page r ange & $522-525$ \\
\hline year & 2014 10-03 \\
\hline URL & ht t p: //hdl . handl e. net /2297/40595 \\
\hline
\end{tabular}




\title{
Phase-Shift Extraction from Twice-Normalized Light Intensity Changes Recorded with Random Phase Shifts
}

\author{
Masaaki Adachi* and Satoshi Sunada \\ Institute of Science and Engineering, Kanazawa University, Kakuma-machi, \\ Kanazawa-shi, Ishikawa 920-1192, Japan
}

A simple phase-shift extraction algorithm is proposed for interferograms recorded with random phase shifts that vary over at least $2 \pi$. The phase-shift-dependent changes in the intensity at two pixels having different phases, selected from one frame, are taken out and normalized. The sum and difference of the two normalized changes are calculated, and both the changes are normalized again along the phase shifts. The normalized sum and difference become the cosine and sine of a term including the random phase shift, respectively. Thereby, the phase shifts are extracted from both twice-normalized intensity changes. An experiment using an interference microscope equipped with a piezoelectric-transducer positioner of an objective lens is conducted to estimate the validity of the algorithm. The algorithm is verified to have satisfactory results when the multiple interferograms used have a sample size of more than 15 frames recorded with random phase shifts.

Keywords: phase shift, phase extraction, random phase shifts, interferometry, vertical vibration

*E-mail: adachi@staff.kanazawa-u.ac.jp 


\section{Introduction}

Phase shifting is one of the most powerful methods in interferometry, and a phase distribution is accurately extracted when phase shifts are precisely conducted. However, a phase shifter generally has nonlinear response and a small vertical vibration would disturb phase shifts. Therefore, a number of phase-shift algorithms ${ }^{1)}$ have been developed to be less sensitive to phase-shift errors. However, even in well-designed phase-shift algorithms, the accuracy is heavily dependent on the errors in the phase shift. This means that phase-shifting techniques are difficult to apply in vertically vibrating environments where the actual phase shifts differ substantially from the desired amount. In such a situation, if the actual phase shifts can be determined, then the phase distribution can be extracted using a least-squares algorithm. ${ }^{2,3)}$ Thus, a phase-shift extraction algorithm using recorded interferograms has been highly desired. For many different approaches of estimating an unknown phase shift, Larkin ${ }^{4}$ has reviewed and proposed an estimation using vortex transform. His method can work using three or more interferograms recorded with random phase shifts, but his concept, combining temporal and spatial methods, is complex and only computer simulations are presented, with no experimental data. Patil and Rastogi ${ }^{5)}$ have also reviewed and proposed a stochastic algorithm that characterizes the nonlinear response of the phase shifter. Referring back to the random phase shifts, it has been difficult to extract phase shifts with a simple technique.

Recently, simple phase-shift extraction techniques using a series of interferograms recorded with random phase shifts have been proposed by $\mathrm{Xu}$ et al., ${ }^{6)}$ Hao et al., ${ }^{7)}$ and $\mathrm{Xu}$ et al. ${ }^{8)}$ Their techniques utilize an arccosine operation that calculates only the absolute value of the phase. The unknown sign of the phase should be determined with statistical, histogram, or temporal information of the phase. Therefore, the extraction processes are not straightforward and require a substantial computational load. 
In this paper, we propose a simple phase-shift extraction algorithm that does not use the arccosine operation. The algorithm is designed for multiple interferograms recorded with random phase shifts. Two pixels of different phases are selected in one frame, and the algorithm searches for the maximum and minimum light intensities at these pixels in all recorded frames. These intensities are then used to normalize the intensity changes at the pixels. The sum and difference of the normalized changes are calculated and then are normalized again. Both twice-normalized changes are used to extract the phase shifts. The extraction is conducted with any two pixels with different phases. Thus, it could be applied to not only a smooth surface but also a rough surface. In addition, as there are many different combinations of two pixels in a frame, many phase shifts are extracted and could be used to approach a more accurate phase shift having a unique amount.

It is well known that normalizing the intensity change can easily remove a background and induce a constant modulation of the fringe pattern. Chen et al. ${ }^{9}$ ) have utilized such a technique of normalizing the intensity change to extract the phase distribution. Their algorithm normalizes light intensities and calculates the sum and difference of the normalized intensities. After the calculations, the extreme values of these quantities are searched and used to extract the phase difference between pixels, because they thought that the difference between the two extreme values is linked to the correlation of light intensity changes at pixels. The clear differences from our algorithm are that the sum and difference of the normalized changes are not normalized again, and the obtained results indicate phase distributions that differ in physical quantities from ours.

\section{Principle}

In our algorithm, multiple interferograms are supposed to be successively recorded with random phase shifts varying over at least $2 \pi$. A random phase shift in the $k$ th recorded frame with respect to the first frame is denoted as $\delta_{\mathrm{k}}$, and the intensity $I_{\mathrm{k}}$ at pixel $(x, y)$ in this frame is given by 


$$
I_{k}(x, y)=I_{b}(x, y)+I_{m}(x, y) \cos \left[\phi(x, y)+\delta_{k}\right],
$$

where $I_{\mathrm{b}}(x, y)$ is the background intensity, $I_{\mathrm{m}}(x, y)$ is the modulation intensity, and $\phi(x, y)$ is the phase. For two different pixel positions, labeled $\mathrm{A}$ and $\mathrm{B}$, in the frame, the algorithm searches over all phase shifts for the maximum and minimum intensities. The normalized intensity changes at both A and B, namely, $N I_{\mathrm{k}}(\mathrm{A})$ and $N I_{\mathrm{k}}(\mathrm{B})$, respectively, are expressed as

$$
\begin{gathered}
N I_{k}(A)=\cos \left[\phi(A)+\delta_{k}\right], \\
N I_{k}(B)=\cos \left[\phi(B)+\delta_{k}\right] .
\end{gathered}
$$

The sum and difference of these normalized changes are calculated as

$$
\begin{aligned}
& N I_{k}(A)+N I_{k}(B)=\cos \left[\phi(A)+\delta_{k}\right]+\cos \left[\phi(B)+\delta_{k}\right] \\
& =2 \cos \left[\frac{\phi(A)+\phi(B)+2 \delta_{k}}{2}\right] \cdot \cos \left[\frac{\phi(A)-\phi(B)}{2}\right], \\
& N I_{k}(A)-N I_{k}(B)=\cos \left[\phi(A)+\delta_{k}\right]-\cos \left[\phi(B)+\delta_{k}\right] \\
& =-2 \sin \left[\frac{\phi(A)+\phi(B)+2 \delta_{k}}{2}\right] \cdot \sin \left[\frac{\phi(A)-\phi(B)}{2}\right] .
\end{aligned}
$$

The algorithm then searches for the maximum and minimum values for the normalized sum and difference, and then normalizes these changes again. The twice-normalized changes $T N I_{\mathrm{k}}{ }^{\mathrm{SUM}}$ and $T N I_{\mathrm{k}} \mathrm{DIF}$ are

$$
T N I_{k}^{S U M}=\cos \left[\frac{\phi(A)+\phi(B)+2 \delta_{k}}{2}\right],
$$




$$
T N I_{k}^{D I F}=-\sin \left[\frac{\phi(A)+\phi(B)+2 \delta_{k}}{2}\right]
$$

These changes have the same argument including the desired phase shift $\delta_{\mathrm{k}}$. Therefore, the phase shift between the first frame and the kth frame, $\delta_{\mathrm{k}}-\delta_{0}=\delta_{\mathrm{k}}$, is given by

$$
\delta_{k}=\arg \left(T N I_{k}^{S U M}-i \cdot T N I_{k}^{D I F}\right)-\arg \left(T N I_{0}^{S U N}-i \cdot T N I_{0}^{D I F}\right),
$$

where $i$ is the imaginary unit.

The accuracy of the extracted phase shift will depend on the accuracy of the two normalizations. The first normalization depends on the number of interferograms, and a small sample size might increase the error in determining the maximum and minimum intensities. This error will be considered in Discussion. The second normalization depends on the phase difference between A and B. If the second cosine term in eq. (4) or the second sine term in eq. (5) is small, then the second normalization will amplify the unavoidable intensity noise. However, the difference between the phases of A and B can be controlled by a simple selection process for B as shown in the following section.

\section{Experimental Methods}

To verify the algorithm, we conducted an experiment using an interference microscope and unfiltered light from an amber light-emitting diode. A silicon wafer with a crack at the center was used as the object. Interferograms were recorded using a Gigabit Ethernet charge-coupled device camera with a 12-bit analog-to-digital converter and were transferred to a computer for data processing. A piezoelectric transducer (PZT) lens positioner, whose extension was controlled by the analog random voltage generated by another computer with 800 $\mathrm{Hz}$, was used to produce random phase shifts during data collection. A total of 41 interferograms were recorded (the frame index $k$ runs from 0 to 40). 
The intensity in a $300 \times 300$ pixel area around the center of the first frame $(k=$ 0 ) is shown in Fig. 1. The intensity distribution of the upper line, $y=300$, is collected from all recorded frames, and the absolute difference between the maximum and minimum intensities is calculated at each $x$ pixel. The pixel along this line with the largest difference is set as A. Then, we find a frame $k$ for which the intensity at $\mathrm{A}$ is around the average of the maximum and minimum intensities. In the found frame having $k=7$, the pixel in the line with the maximum intensity is set as $B$. The result of this is that $\cos \{[\phi(A)-\phi(B)] / 2\}$ in eq. (4) and $\sin \{[\phi(A)$ $-\phi(B)] / 2\}$ in eq. (5) have nearly the same absolute value and both are far from zero. The intensity changes at A and B for 40 random phase shifts are displayed in Fig. 2, and these are used to calculate $\delta_{\mathrm{k}}$ using eq. (8). As the two-pixel searches and phase-shift calculation can be performed for any line in the frame, this search and calculation are repeated for all lines in the interferogram frame to estimate the accuracy of phase-shift calculation.

The phase-shift patterns for some lines are very similar, but other lines have patterns with opposite signs. This difference arises from the sign of the second term in eq. (5): for some lines, $\phi(\mathrm{A})$ is greater than $\phi(\mathrm{B})$, and for other lines, $\phi(\mathrm{A})$ is less than $\phi(B)$. Therefore, the signs of the calculated phase shifts in lines $y<$ 300 are reversed to agree with that in line $y=300$. When a PZT is used to shift the phase, the phase change could be positive for the expansion of the PZT is positive. In our experiment, the first phase shift due to vibrations does not mean a positive expansion; thus, the sign of the first phase shift is not decided.

The obtained phase shifts $\delta_{\mathrm{k}}$ and the difference $I_{S \mathrm{~d}}$ between the maximum and minimum intensities at $\mathrm{A}$ are used to calculate $Z_{\mathrm{k}}=I_{S \mathrm{~d}} \cdot \exp \left(i \delta_{\mathrm{k}}\right)$. Figure 3 shows the values of $Z_{4}$ in all 300 lines of the 4 th interferogram frame. The phase shifts take very similar values and their histogram has a Gaussian-like shape with a standard deviation of $0.06 \mathrm{rad}$. This indicates that the deviation is a random property and appears to originate mainly from air disturbance and digitization errors, and then a high-precision phase shift could be approached by averaging. 
$0.0035 \mathrm{rad}$. The mean phase shifts and standard deviations of the mean in all frames are shown in Fig. 4. We then used the mean phase shifts and a leastsquares algorithm $\left.{ }^{2}\right)$ to extract the phase distribution of the interferogram in Fig. 1. The phase distribution is shown in Fig. 5, and the phase distribution along the line $y=60$ is shown in Fig. 6. As the silicon wafer surface is flat at the position defined by the $y=60$ line, the linear change in the phase confirms that the extracted mean phase shifts are correct. The above shift extractions at all the lines and averaging them lead to a high-precision shift, but consume more calculation time. Our calculation to obtain the final shift takes $38 \mathrm{~ms}$ with a quad-core cpu and LabVIEW programing language. This time is $1 / 35$ times the least-squares calculation time for the phase distribution in Fig. 5.

\section{Discussion}

Let us consider here the accuracy and resolution of this algorithm. As mentioned in the principle, the performance of the first normalization depends on the number of interferograms, and a small sample size might underestimate the modulation intensity compared with the true value. However, if underestimation ratios are the same between $\mathrm{A}$ and $\mathrm{B}$, both the sum and the difference decrease. Thus, the second normalization results in the absence of error. When underestimation ratios are different between $\mathrm{A}$ and $\mathrm{B}$, the $\delta_{\mathrm{k}}$ given in eq. (8) might have a significant error. To estimate this error, the mean standard deviation of $\delta_{\mathrm{k}}$ is calculated from a smaller sample size of interferograms recorded. Figure 7 shows the standard deviation and the mean of $\delta_{4}$ in the sample size range from 5 to 41 . The sample size of 15 is estimated to be sufficient for obtaining the same deviation as in Fig. 3. In the sample size range from 14 to 6 , the mean takes the same value as that of 15 , but the standard deviation increases. Recording sample sizes of more than 15 is not so difficult as high-speed and high-sensitivity cameras are currently available. The use of such cameras easily reduces these errors. It should be noted that the mentioned sample size depends on the amplitude of random phase shifts. The interferograms used are recorded with phase shifts that vary over at least $2 \pi$. 
In terms of the resolving power of this technique, a large number of possible $\mathrm{A}$ and $\mathrm{B}$ pixel combinations in the recorded area will reduce the standard deviation of the mean phase shift. This high resolution originates from the fact that the extracted phase shifts can be obtained from the change in the intensity at only two positions in the interferogram and no spatial information is supposed. This implies that the proposed algorithm is also applicable to speckle interferograms.

\section{Conclusions}

We have proposed a simple phase-shift extraction algorithm for interferograms recorded with random phase shifts. The algorithm selects two pixels and normalizes their intensity changes. The sum and difference of the two normalized changes are calculated and normalized again. The phase shifts are extracted from both twice-normalized intensity changes. The extracted phase shifts can accurately calculate the phase distribution.

\section{Acknowledgment}

This work was supported by JSPS KAKEN Grant Number 24560289. 


\section{References}

1. H. Schreiber and J. H. Bruning: in Optical Shop Testing, ed. D. Malacara (Wiley, 2007) 3rd ed., Chap. 14, p. 547.

2. J. E. Greivenkamp: Opt. Eng. 23 (1984) 350.

3. G. Lai and T. Yatagai: J. Opt. Soc. Am. A 8 (1991) 822.

4. K. G. Larkin: Opt. Express 9 (2001) 236.

5. A. Patil and P. Rastogi: Opt. Lasers Eng. 43 (2005) 457.

6. X. F. Xu, L. Z. Cai, Y. R. Wang, X. L. Yang, X. F. Meng, G. Y. Dong, X. X. Shen, and H. Zhang: Appl. Phys. Lett. 90 (2007) 121124.

7. Q. Hao, Q. Zhu, and Y. Hu: Opt. Lett. 34 (2009) 1288.

8. J. Xu, Y. Li, H. Wang, L. Chai, and Q. Xu: Opt. Exp. 18 (2010) 24368.

9. X. Chen, M. Gramaglia, and J. A. Yeazell: Appl. Opt. 39 (2000) 585. 


\section{Figure Captions}

Fig. 1. Interferogram frame obtained by an interference microscope equipped with a $5 \times$ objective lens. The object is a silicon wafer with a crack at the center.

Fig. 2. Intensity changes at positions $\mathrm{A}$ and $\mathrm{B}$ as a function of the frame index $k$ taken from interferograms recorded with random phase shifts that vary over at least $2 \pi$. At the frame of $k=7$, the intensity at $\mathrm{A}$ is around the average of the maximum and minimum intensities, and the intensity at $\mathrm{B}$ has the maximum intensity.

Fig. 3. Polar plot of $Z_{4}=I_{S \mathrm{~d}} \cdot \exp \left(i \delta_{4}\right)$. The intensity $I_{S \mathrm{~d}}$ is given in arbitrary units. Data from all the horizontal lines in the interferogram frame in Fig. 1, including the crack area, are included in this plot. The standard deviation of the shifts is $0.06 \mathrm{rad}$.

Fig. 4. Extracted mean phase shifts and standard deviations of the mean as a function of the frame index.

Fig. 5. Phase distribution of the interferogram in Fig. 1. This distribution is extracted using the mean phase shifts shown in Fig. 4 and a least-squares $\operatorname{algorithm.~}^{2)}$ 
Fig. 6. Phase distribution along the line $y=60$ in Fig. 5. The silicon wafer on this line is extremely flat. The linear change in the phase distribution demonstrates that the phase-shift extraction algorithm proposed here works correctly.

Fig. 7. Mean phase shift of $\delta_{4}$ and its standard deviation along the sample size of frames. A size of more than 15 is sufficient for obtaining an accurate phase shift in our experiment. 


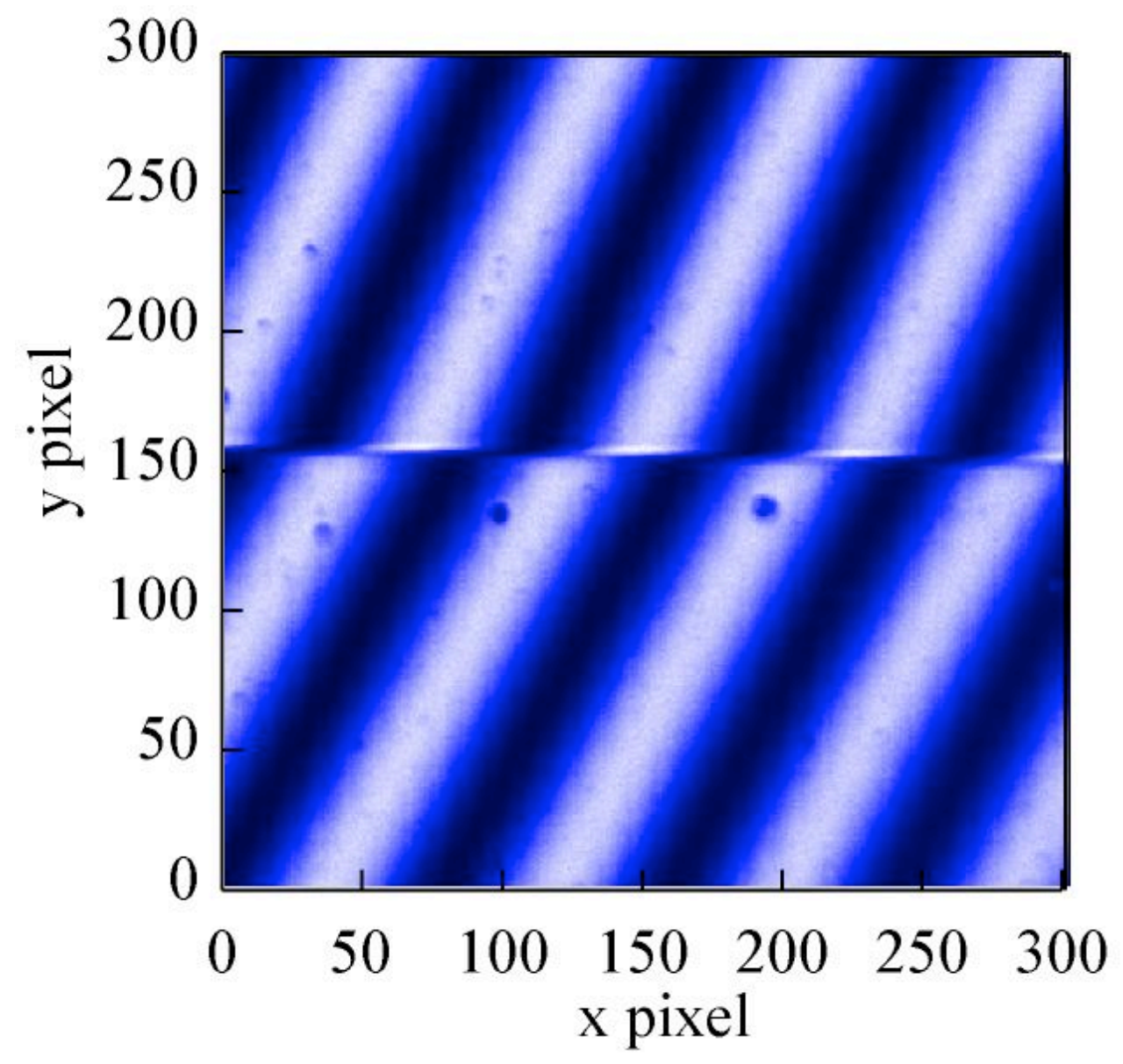

Figure 1 


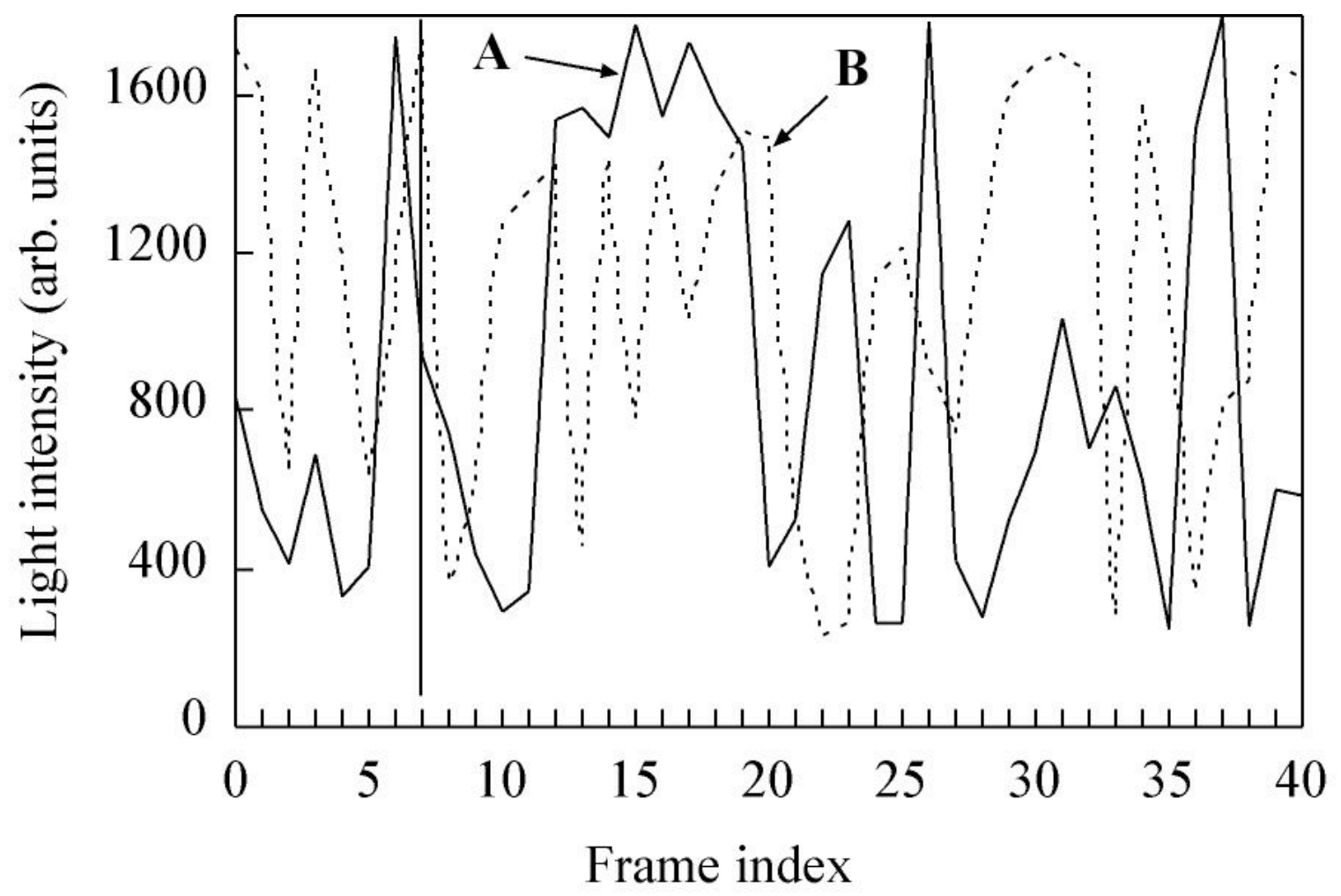

Figure 2 


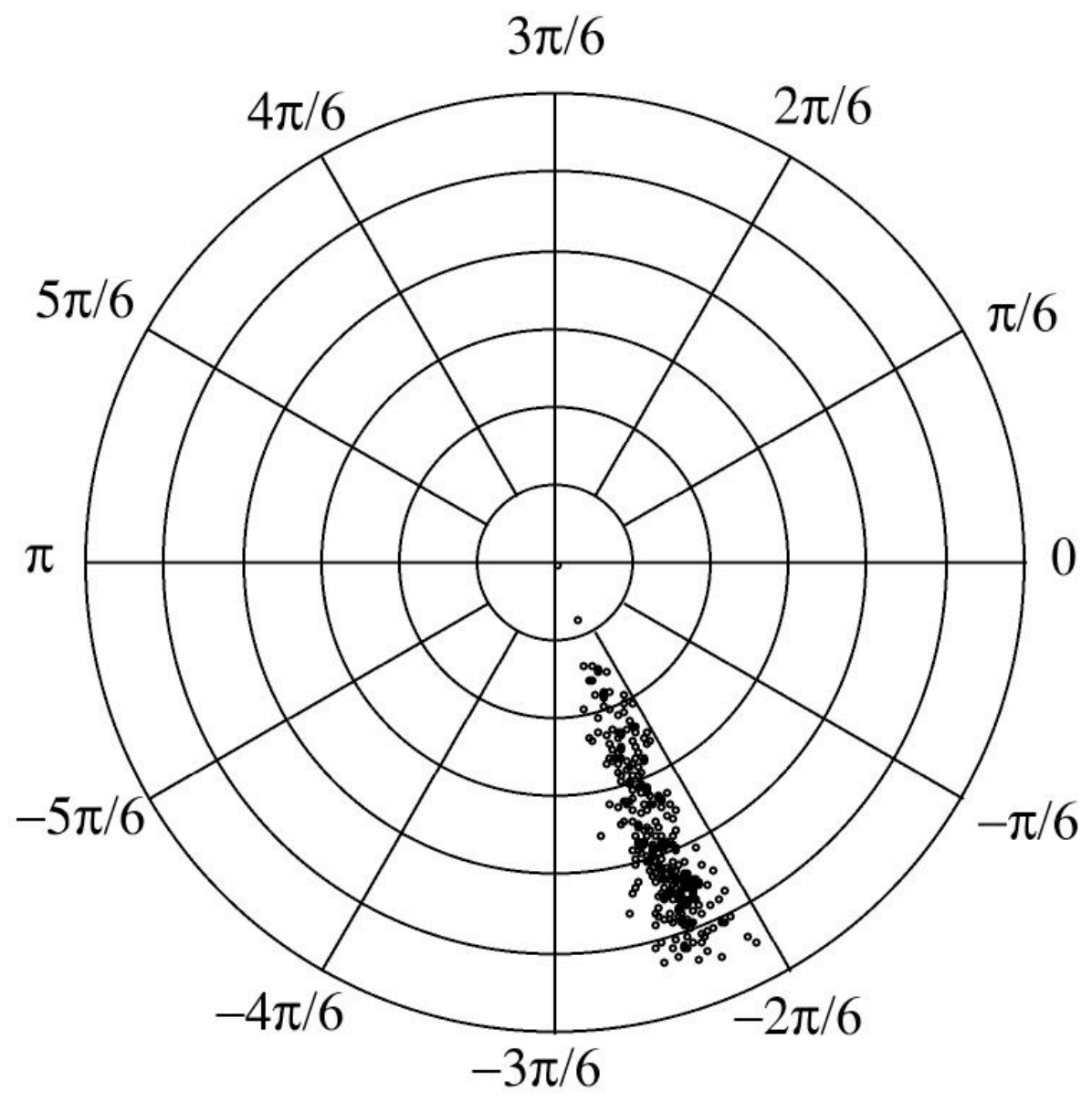

Figure 3 


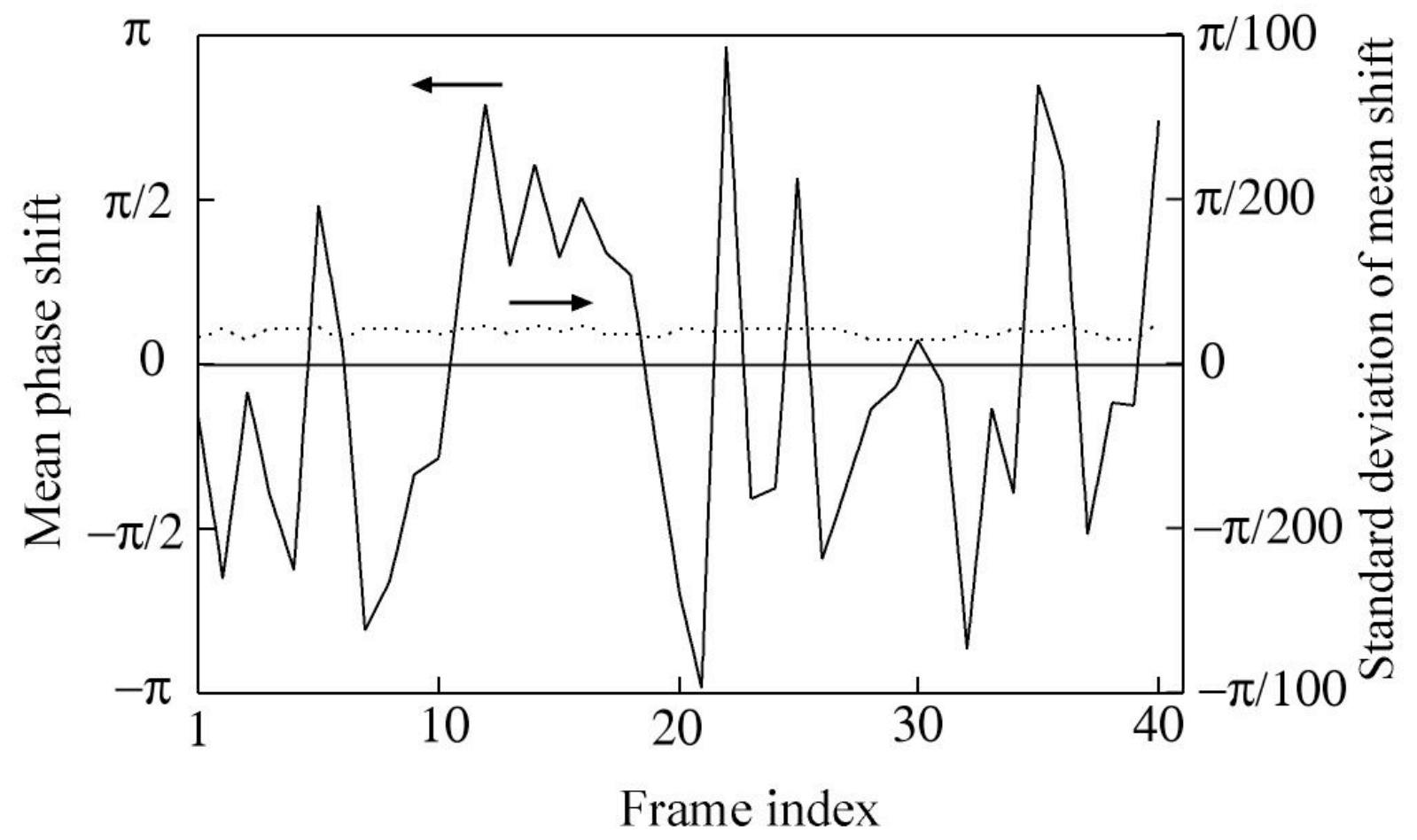

Figure 4 


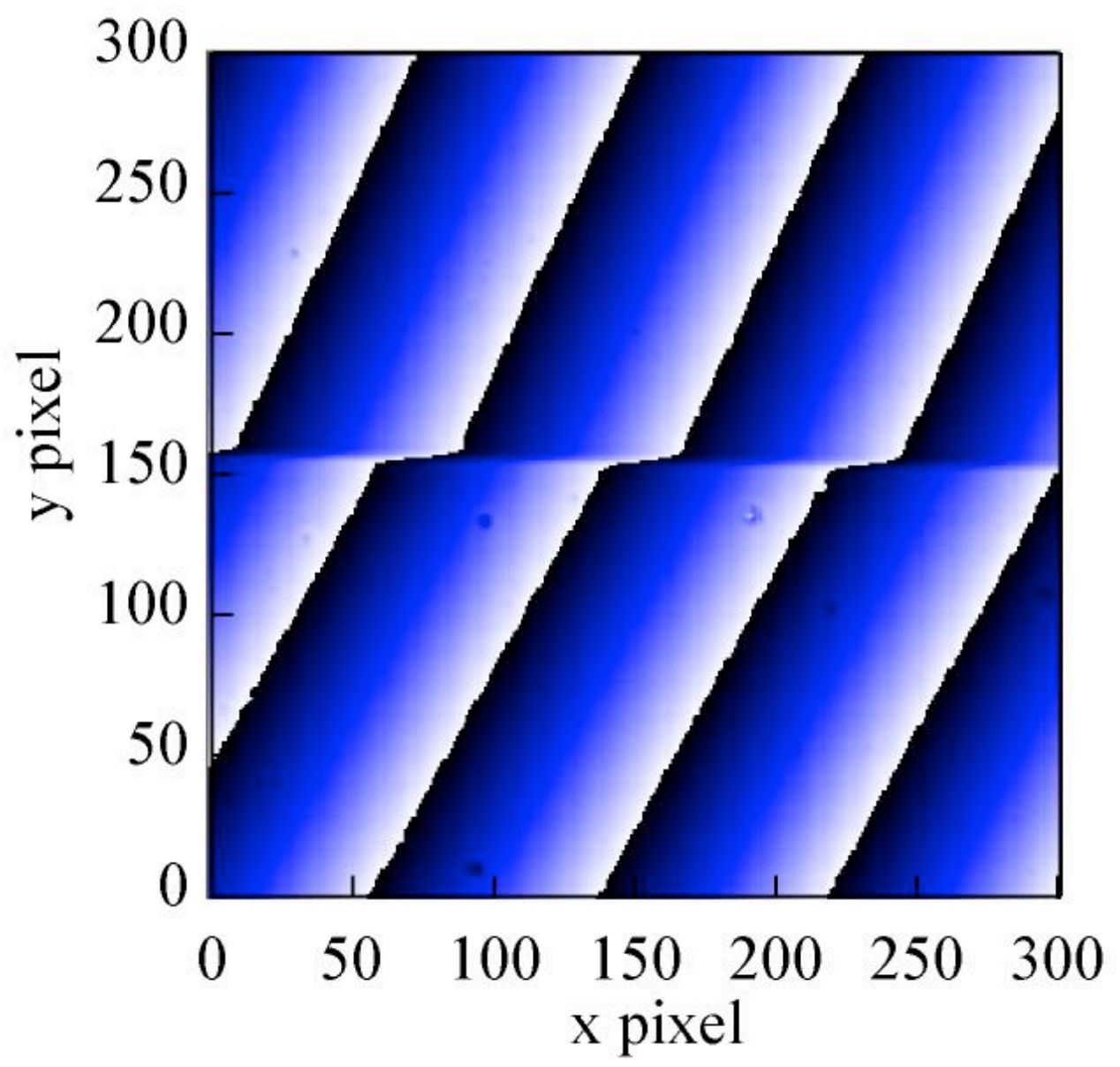

Figure 5 


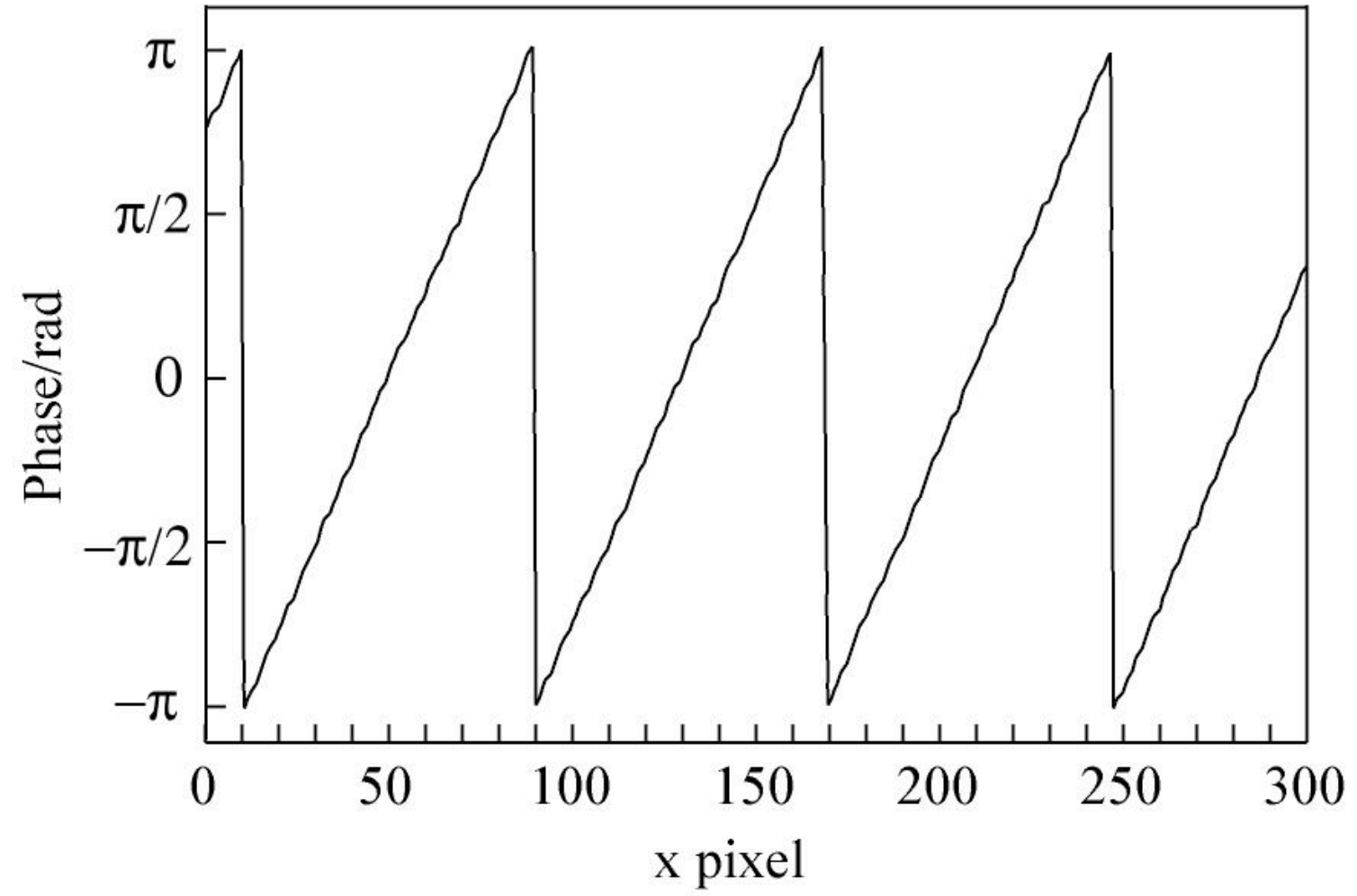

Figure 6 


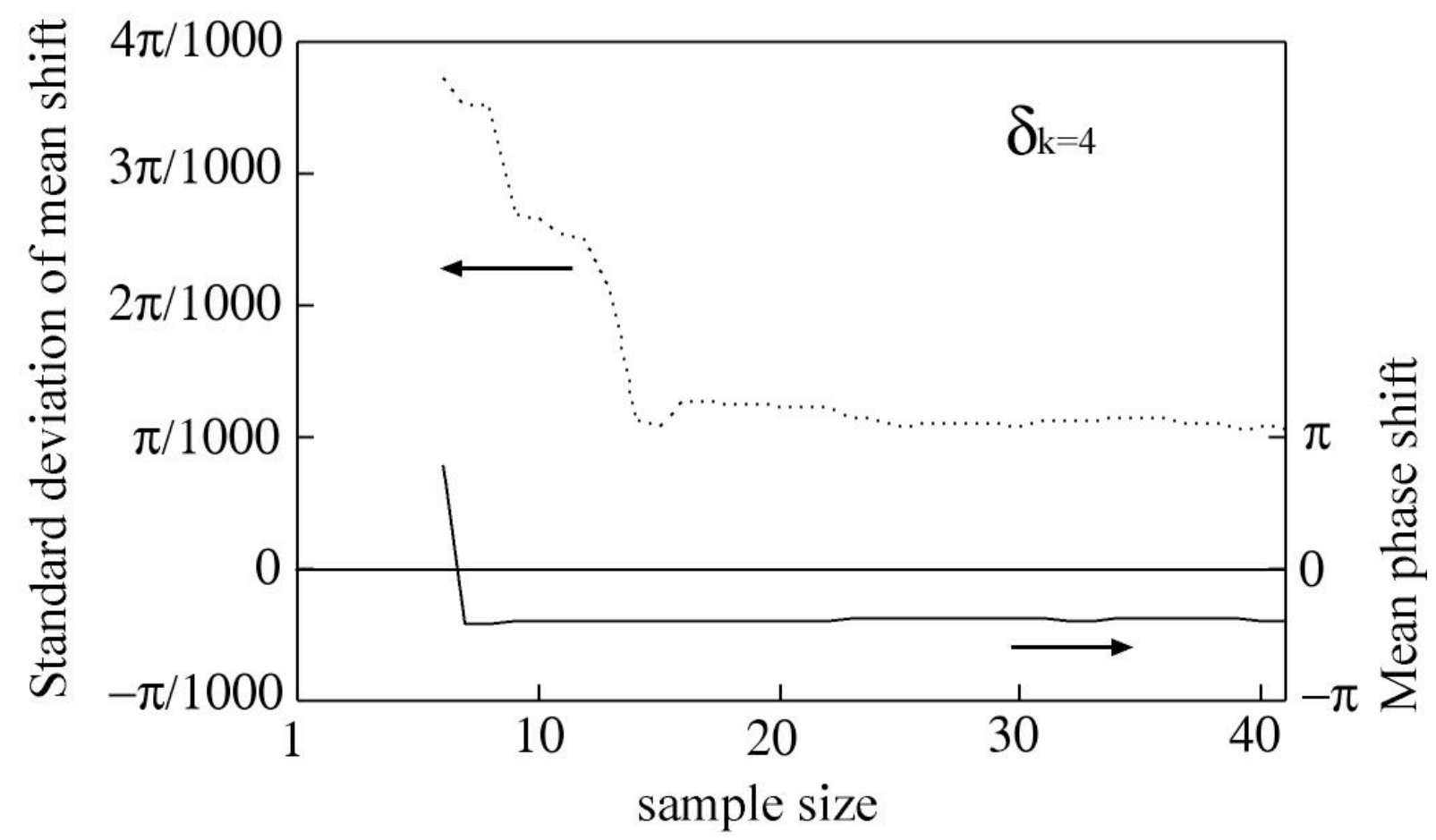

Figure 7 